Homology, Homotopy and Applications, vol.14(2), 2012, pp.23-36

\title{
A REPRESENTABILITY THEOREM FOR SOME HUGE ABELIAN CATEGORIES
}

\section{GEORGE CIPRIAN MODOI}

\author{
(communicated by Charles A. Weibel)
}

\begin{abstract}
We define quasi-locally presentable categories as big unions of a chain of coreflective subcategories that are locally presentable. Under appropriate hypotheses we prove a representability theorem for exact contravariant functors defined on a quasi-locally presentable category taking values in abelian groups. We show that the abelianization of a well generated triangulated category is quasi-locally presentable, and we obtain a new proof of the Brown representability theorem. Examples of functors that are not representable are also given.
\end{abstract}

\section{Introduction}

One of the main problems occurring in the theory of triangulated categories is to construct a left or right adjoint for a given triangulated functor. In his influential book on this subject, Neeman shows that the problem of finding an adjoint for a functor between triangulated categories may be equivalently studied at the level of abelianizations of these categories, where we have to construct an adjoint for some exact functor between abelian categories (see [11, Proposition 5.3.9]). Further, Neeman considers in [11, Remark 5.3.10] that, unfortunately, this idea is "nearly impossible" to be applied, since "existence theorems of adjoints usually depend on the categories being well-powered", that is, one object must have only a set of subobjects (for an object of an abelian category this it equivalent to having only a set of quotients). But, in general, the abelianization of a triangulated category with arbitrary coproducts is huge, that is, it does not satisfy the condition of being well (co)powered; see [11, Appendix C]. Hence the abelianization is often considered to be too big and thus not manageable. (See also the Introduction of Krause's work [8].) This paper intends to change this perspective a little. More exactly, the result about the existence of adjoints depending on the categories being well powered is, obviously, the special Freyd's adjoint functor theorem: if $\mathcal{C}$ is a complete, well powered category having a cogenerator, then every functor $F: \mathcal{C} \rightarrow \mathcal{D}$ has a left adjoint if and only

Research supported by CNCS-UEFISCDI grant PN-II-RU-TE-2011-3-0065.

Received January 20, 2011, revised November 7, 2011, March 13, 2012, May 8, 2012; published on October 4, 2012.

2000 Mathematics Subject Classification: 18A40, 18E30.

Key words and phrases: representable functor, quasi-locally presentable category, abelianization, triangulated category with coproducts.

Article available at http://intlpress.com/HHA/v14/n2/a2 and doi:10.4310/HHA.2012.v14.n2.a2

Copyright (C) 2012, International Press. Permission to copy for private use granted. 
if it preserves limits; see [4, p. 89]. We argue that even if the abelianization of a well generated triangulated category is not always well (co)powered, it has enough structure to allow us to apply the general Freyd's adjoint functor theorem: if $\mathcal{C}$ is a complete category, then every functor $F: \mathcal{C} \rightarrow \mathcal{D}$ has a left adjoint if and only if it preserves limits and satisfies the solution set condition (that is, for every $f: D \in \mathcal{D}$ there is a set of maps $f_{i}: D \rightarrow F\left(C_{i}\right), i \in I$ in $\mathcal{D}$, where $C_{i} \in \mathcal{C}$, such that every map $D \rightarrow F(C)$, with $C \in \mathcal{C}$, factors as $f=F(k) f_{i}$, for some $k: C_{i} \rightarrow C$ in $\mathcal{C}$; see $\left.[\mathbf{1}, 0.7]\right)$. The problem of the existence of the adjoints and the one of representability of a given functor are strongly related (to fix the settings, suppose that we work with preadditive categories): First, a functor $F: \mathcal{C} \rightarrow \mathcal{D}$ has a left adjoint if and only if the functor $\mathcal{D}(D, F(-)): \mathcal{C} \rightarrow \mathcal{A} b$ is representable for all $D \in \mathcal{D}$. Second, a functor $F: \mathcal{C} \rightarrow \mathcal{A} b$ has a left adjoint if and only if it is representable (actually it is represented by the left adjoint evaluated at $\mathbb{Z}$; see [4, pp. 81-82]).

The paper is organized as follows: In the first section we introduce the notion of quasi-locally presentable category; it is a category that may be written as a union of a chain of coreflective subcategories that are locally $\lambda$-presentable, where $\lambda$ runs over all regular cardinals. Under appropriate hypotheses, we prove a representability theorem for exact, contravariant functors defined on such categories.

In the second section we recall the definition of the abelianization of a triangulated category, and we show how the study of Brown representability may be done at the level of this abelianization. For well generated triangulated categories we show that the abelianization is quasi-locally presentable and satisfies the supplementary hypotheses allowing us to apply the representability theorem proved in the previous section. As a consequence we obtain a new proof of Brown representability theorem for well generated triangulated categories.

All categories that we work with are preadditive (enriched over $\mathcal{A} b$ ). Everywhere in our paper we may equally adopt the point of view of Gödel-Bernays-Von Neumann axiomatization of set theory, with the distinction made there between classes and sets or to work in a given Grothendieck universe. In this last case, a set means a small set relative to that universe, whereas a class is a set that is not necessarily small.

\section{Acknowledgements}

For the second and third versions of this paper we acknowledge the financial support of the grant CNCS-UEFISCDI code PN-II-RU-TE-2011-3-0065. We also would like to thank an anonymous referee for many suggestions of improvement of the earlier version.

\section{Quasi-locally presentable abelian categories}

We begin this section by recalling some definitions: A cardinal $\lambda$ is said to be regular provided that it is infinite, and it cannot be written as a sum of less than $\lambda$ cardinals, all smaller than $\lambda$. Denote by $\mathfrak{R}$ the class of all regular cardinals.

Let $\mathcal{A}$ be an additive category and $\mathcal{C} \subseteq \mathcal{A}$ be a subcategory. Let $F: \mathcal{A} \rightarrow \mathcal{A} b$ be a contravariant functor. The category of elements of $\left.F\right|_{\mathcal{C}}$, where $\left.F\right|_{\mathcal{C}}$ denotes the 
restriction of $F$ at $\mathcal{C}$, is by definition constructed as follows:

$$
\mathcal{C} / F=\{(X, x) \mid X \in \mathcal{C}, x \in F(C)\},
$$

with the morphisms

$$
\mathcal{C} / F\left(\left(X_{1}, x_{1}\right),\left(X_{2}, x_{2}\right)\right)=\left\{\alpha \in \mathcal{C}\left(X_{1}, X_{2}\right) \mid F(\alpha)\left(x_{2}\right)=x_{1}\right\} .
$$

In particular, for any object $A \in \mathcal{A}$, let

$$
\begin{gathered}
\mathcal{C} / A=\mathcal{C} / \mathcal{A}(-, A)=\{(C, \xi) \mid C \in \mathcal{C}, \xi: C \rightarrow A\}, \\
\mathcal{C} / A\left(\left(C_{1}, \xi_{1}\right),\left(C_{2}, \xi_{2}\right)\right)=\left\{\alpha \in \mathcal{C}\left(C_{1}, C_{2}\right) \mid \xi_{2} \alpha=\xi_{1}\right\} .
\end{gathered}
$$

Consider a regular cardinal $\lambda$. A non-empty category $\mathcal{S}$ is called $\lambda$-filtered if the following two conditions are satisfied:

F1. For every set $\left\{s_{i} \mid i \in I\right\}$ of less that $\lambda$ objects of $\mathcal{S}$, there are an object $s \in \mathcal{S}$ and morphisms $s_{i} \rightarrow s$ in $\mathcal{S}$, for all $i \in I$.

F2. For every set $\left\{\sigma_{i}: s \rightarrow t \mid i \in I\right\}$ of less that $\lambda$ morphisms in $\mathcal{S}$, there is a morphism $\tau: t \rightarrow u$ such that $\tau \sigma_{i}=\tau \sigma_{j}$, for all $i, j \in I$.

Let $A$ be an object of a category $\mathcal{A}$. Then the functor $\mathcal{A}(A,-)$ preserves the colimit of a diagram $\mathcal{S} \rightarrow \mathcal{A}, s \mapsto X(s)$ in $\mathcal{A}$ (indexed over a category $\mathcal{S}$ ), if and only if every map $g: A \rightarrow \operatorname{colim}_{s \in \mathcal{S}} X(s)$ factors as

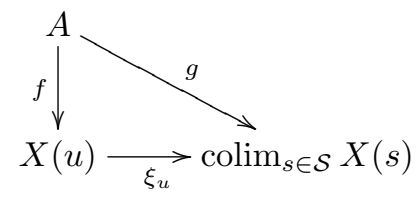

through some of the canonical maps $\xi_{u}$ with $u \in \mathcal{S}$, and every such factorization is essentially unique, in the sense that if $f_{1}, f_{2}: A \rightarrow X(u)$ with $\xi_{u} f_{1}=g=\xi_{u} f_{2}$, then there is $\sigma: u \rightarrow t$ a map in $\mathcal{S}$ such that $X(\sigma) f_{1}=X(\sigma) f_{2}$. The object $A \in \mathcal{A}$ is called $\lambda$-presentable if $\mathcal{A}(A,-)$ preserves all $\lambda$-filtered colimits. The category $\mathcal{A}$ is called locally $\lambda$-presentable provided that it is cocomplete and has a set $\mathcal{S}$ of $\lambda$-presentable objects such that every $X \in \mathcal{A}$ is a $\lambda$-filtered colimit of objects in $\mathcal{S}$ (see $[\mathbf{1}$, Definition 1.17], but also [1, Remark 1.21])). Note that, if $\mathcal{A}$ is locally $\lambda$-presentable, then the subcategory $\mathcal{A}^{\lambda}$ of all $\lambda$-presentable objects in $\mathcal{A}$ is essentially small, and for every object $A \in \mathcal{A}$, the category $\mathcal{A}^{\lambda} / A$ is $\lambda$-filtered and

$$
A \cong \operatorname{colim}_{(X, \xi) \in \mathcal{A}^{\lambda} / A} X
$$

as we may see from [1, Proposition 1.22]. A category is called locally presentable if it is locally $\lambda$-presentable for some regular cardinal $\lambda$.

Remark 1.1. Let $\mathcal{A}$ be a locally $\lambda$-presentable category. Observe then that the category $\mathcal{A}^{\text {op }}$ satisfies the hypotheses of Freyd's special adjoint functor theorem: it is well powered, complete and has a cogenerator (since the coproduct of all $\lambda$-presentable objects is a generator for $\mathcal{A}$ ). In particular, every contravariant functor $F: \mathcal{A} \rightarrow \mathcal{A} b$ that sends colimits into limits is representable. Indeed, we can view $F$ as a covariant functor $\mathcal{A}^{\text {op }} \rightarrow \mathcal{A} b$, which must be representable, having a left adjoint. Let us write 
$F \cong \mathcal{A}(-, A)$ for some $A \in \mathcal{A}$. Thus the categories $\mathcal{A}^{\lambda} / A$ and $\mathcal{A}^{\lambda} / F$ are isomorphic, so

$$
F \cong \mathcal{A}\left(-, \operatorname{colim}_{(X, x) \in \mathcal{A}^{\lambda} / F} X\right)
$$

We consider a category $\mathcal{A}$ that is a union

$$
\mathcal{A}=\bigcup_{\lambda \in \mathfrak{R}} \mathcal{A}_{\lambda}
$$

of a chain of subcategories $\left\{\mathcal{A}_{\lambda} \mid \lambda \in \mathfrak{R}\right\}$ such that $\mathcal{A}_{\kappa} \subseteq \mathcal{A}_{\lambda}$ for all $\kappa \leqslant \lambda$ and the subcategory $\mathcal{A}_{\lambda}$ locally $\lambda$-presentable and closed under colimits in $\mathcal{A}$, for any $\lambda \in \mathfrak{R}$. Denote by $I_{\lambda}: \mathcal{A}_{\lambda} \rightarrow \mathcal{A}$ the inclusion functor Note that by Freyd's special adjoint functor theorem, the subcategory $\mathcal{A}_{\lambda}$ is coreflective, that is, $I_{\lambda}$ has a right adjoint $R_{\lambda}: \mathcal{A} \rightarrow \mathcal{A}_{\lambda}$. We call quasi-locally presentable a category $\mathcal{A}$ as above satisfying the additional property that $R_{\lambda}$ preserves colimits for all $\lambda \in \mathfrak{R}$. For such a quasi-locally presentable category $\mathcal{A}$ and a regular cardinal $\lambda$, we denote by $\mathcal{A}_{\lambda}^{\lambda}$ the subcategory of all $\lambda$-presentable objects of $\mathcal{A}_{\lambda}$ that has to be skeletally small.

Lemma 1.2. In a quasi-locally presentable category $\mathcal{A}$, it holds $\mathcal{A}_{\kappa}^{\kappa} \subseteq \mathcal{A}_{\lambda}^{\lambda}$, for every $\kappa \leqslant \lambda$.

Proof. With the notations above, fix two cardinals $\kappa \leqslant \lambda$. Observe that if we denote $I_{\kappa, \lambda}: \mathcal{A}_{\kappa} \rightarrow \mathcal{A}_{\lambda}$ the inclusion functor, then it has a right adjoint namely $R_{\kappa, \lambda}=R_{\kappa} I_{\lambda}$. Since $R_{\kappa}$ preserves colimits, $R_{\kappa, \lambda}$ satisfies the same property. Then for $A \in \mathcal{A}_{\kappa}^{\kappa}$ and for a $\lambda$-filtered (hence also $\kappa$-filtered) diagram $\left(X_{i}\right)_{i \in I}$ in $\mathcal{A}_{\lambda}$ we have the following chain of isomorphisms, showing that $I_{\kappa, \lambda}(A)$ is $\lambda$-presentable:

$$
\begin{aligned}
\mathcal{A}_{\lambda}\left(I_{\kappa, \lambda}(A), \operatorname{colim} X_{i}\right) & \cong \mathcal{A}_{\kappa}\left(A, R_{\kappa, \lambda}\left(\operatorname{colim} X_{i}\right)\right) \cong \mathcal{A}_{\kappa}\left(A, \operatorname{colim} R_{\kappa, \lambda}\left(X_{i}\right)\right) \\
& \cong \operatorname{colim} \mathcal{A}_{\kappa}\left(A, R_{\kappa, \lambda}\left(X_{i}\right)\right) \cong \operatorname{colim} \mathcal{A}_{\lambda}\left(I_{\kappa, \lambda}(A), X_{i}\right) .
\end{aligned}
$$

As an example of quasi-locally presentable categories we mention first the classical locally presentable ones. Clearly if $\mathcal{A}$ is locally $\kappa$-presentable for some regular cardinal $\kappa$, then it is also quasi-locally presentable, for all regular cardinals $\lambda$ putting $\mathcal{A}_{\lambda}=\mathcal{A}$, if $\lambda \geqslant \kappa$, and $\mathcal{A}_{\lambda}=0$ otherwise.

Lemma 1.3. Let $F: \mathcal{A} \rightarrow \mathcal{A} b$ be a contravariant functor that sends colimits into lim$i$ its, defined on a quasi-locally presentable, abelian category $\mathcal{A}$. Then for every regular cardinal $\kappa$, there is $\lambda \in \mathfrak{R}, \lambda \geqslant \kappa$ such that

$$
F I_{\kappa} \cong \operatorname{colim}_{(X, x) \in \mathcal{A}_{\lambda}^{\lambda} / F} \mathcal{A}\left(I_{\kappa}(-), X\right) .
$$

Proof. For any $\lambda \in \mathfrak{R}$, consider the corresponding coreflective locally $\lambda$-presentable subcategory $I_{\lambda}: \mathcal{A}_{\lambda} \leftrightarrows \mathcal{A}: R_{\lambda}$

Fix $\kappa \in \mathfrak{R}$. For a skeleton $\mathcal{C}_{0}$ of $\mathcal{A}_{\kappa}^{\kappa}$, denote $C_{0}=\coprod_{(U, u) \in \mathcal{C}_{0} / F} U$. Let $\lambda$ be a regular cardinal such that

$$
\lambda>\kappa+\operatorname{card} \mathcal{C}_{0}+\sum_{U \in \mathcal{C}_{0}} \operatorname{card} F(U)+\sum_{U \in \mathcal{C}_{0}} \operatorname{card} \mathcal{A}\left(U, C_{0}\right)+\aleph_{1} .
$$


Since $F: \mathcal{A} \rightarrow \mathcal{A} b$ sends colimits into limits, the same property is also true for $F I_{\lambda}: \mathcal{A}_{\lambda} \rightarrow \mathcal{A} b$. By Remark 1.1 we obtain $F I_{\lambda} \cong \mathcal{A}_{\lambda}\left(-, F_{\lambda}\right)$ for some $F_{\lambda} \in \mathcal{A}_{\lambda}$ satisfying

$$
F_{\lambda}=\underset{(X, x) \in \mathcal{A}_{\lambda}^{\lambda} / F}{\operatorname{colim}} X=\underset{(X, \xi) \in \mathcal{A}_{\lambda}^{\lambda} / F_{\lambda}}{\operatorname{colim}} X
$$

with the canonical maps $\gamma_{(X, x)}: X \rightarrow F_{\lambda}$. Note that $\gamma_{(X, x)}$ is the image of $(X, x)$ via the isomorphism of categories $\mathcal{A}_{\lambda}^{\lambda} / F I \lambda \stackrel{\cong}{\longrightarrow} \mathcal{A}_{\lambda}^{\lambda} / F_{\lambda}$.

We have to show that

$$
F(A) \cong \operatorname{colim}_{(X, x) \in \mathcal{A}_{\lambda}^{\lambda} / F} \mathcal{A}(A, X),
$$

for all $A \in \mathcal{A}_{\kappa}$. Since $A=I_{\kappa}(A)=I_{\lambda}(A)$ this means precisely that $\mathcal{A}(A,-)$ preserves the colimit of the diagram $\mathcal{A}_{\lambda}^{\lambda} / F \rightarrow \mathcal{A},(X, x) \mapsto X$. In order to prove this, consider in the first step that $A$ is a coproduct of objects in $\mathcal{A}_{\kappa}^{\kappa}$. Without losing the generality, we may assume that $A=\coprod_{i \in I} U_{i}$, for some set $I$, and some $U_{i} \in \mathcal{C}_{0}$. Denote by $j_{i}: U_{i} \rightarrow$ $A,(i \in I)$ the canonical injections. Let $g: A \rightarrow F_{\lambda}$ be a map in $\mathcal{A}$. Since for all $U \in \mathcal{C}_{0}$ we have $U \in \mathcal{A}_{\kappa} \subseteq \mathcal{A}_{\lambda}$, we may identify $\mathcal{C}_{0} / F$ with $\mathcal{C}_{0} / F_{\lambda}$ thus $C_{0}=\coprod_{(U, v) \in \mathcal{C}_{0} / F_{\lambda}} U$ with the canonical injections $\epsilon_{(U, v)}: U \rightarrow C_{0}$. Since $g j_{i} \in \mathcal{A}\left(U_{i}, F_{\lambda}\right)$ we get a unique $f: A \rightarrow C_{0}$, such that $f j_{i}=\epsilon_{\left(U_{i}, g j_{i}\right)}$ from the universal property of the coproduct. Put $c_{0}=(v)_{(U, v) \in \mathcal{C}_{0} / F_{\lambda}}$. We know by Lemma 1.2 that $\mathcal{A}_{\kappa}^{\kappa} \subseteq \mathcal{A}_{\lambda}^{\lambda}$, so the condition $\lambda>\sum_{U \in \mathcal{C}_{0}}$ card $F(U)$ assures us that $\left(C_{0}, c_{0}\right) \in \mathcal{A}_{\lambda}^{\lambda} / F_{\lambda}$. It follows $\left(C_{0}, c_{0}\right) \in \mathcal{A}_{\lambda}^{\lambda} / F$. Moreover, by construction, $\gamma_{\left(C_{0}, c_{0}\right)} f=g$, so $g$ factors through $\gamma_{\left(C_{0}, c_{0}\right)}$.

It remains to show that this factorization is essentially unique. Consider therefore two maps $f_{1}, f_{2}: A \rightarrow C_{0}$ such that $\gamma_{\left(C_{0}, c_{0}\right)} f_{1}=g=\gamma_{\left(C_{0}, c_{0}\right)} f_{2}$. Denote $\mathcal{N}=\{(U, h) \mid$ $U \in \mathcal{C}_{0}, h \in \mathcal{A}\left(U, C_{0}\right)$ with $\left.\gamma_{\left(C_{0}, c_{0}\right)} h=0\right\}$, where $\mathcal{C}$ is a skeleton of $\mathcal{A}_{\lambda}^{\lambda}$ and put $C_{1}=$ $\amalg_{(U, h) \in \mathcal{N}} U$ with the canonical injections $k_{(U, h)}: U \rightarrow C_{1}$. By the choice of $\lambda$ we have $\lambda>\operatorname{card} \mathcal{A}\left(U, C_{0}\right) \geqslant \operatorname{card} \mathcal{N}$, hence $\left(C_{1}, 0\right) \in \mathcal{A}_{\lambda}^{\lambda} / F$. We may even consider $\left(C_{1}, 0\right) \in$ $\mathcal{C} / F$. We have $\left(U_{i},\left(f_{1}-f_{2}\right) j_{i}\right) \in \mathcal{N}$. Hence there is a unique $\theta: A \rightarrow C_{1}$ such that $\theta j_{i}=k_{\left(U_{i},\left(f_{1}-f_{2}\right) j_{i}\right)}$ for all $i \in I$. Further, there is a unique morphism $\eta: C_{1} \rightarrow C_{0}$ such that $\eta k_{(U, h)}=h$ for all $(U, h) \in \mathcal{N}$. Clearly $\eta$ is a map in $\mathcal{A}_{\lambda}^{\lambda} / F$ between $\left(C_{1}, 0\right)$ and $\left(C_{0}, c_{0}\right)$. If $C$ is defined by the exactness of the sequence $C_{1} \stackrel{\eta}{\rightarrow} C_{0} \stackrel{\delta}{\rightarrow} C \rightarrow 0$, then $C \in \mathcal{A}_{\lambda}^{\lambda}$, because $\mathcal{A}_{\lambda}^{\lambda}$ is closed under cokernels (see [1, Proposition 1.16]). Since $F$ sends cokernels into kernels, we infer that there is $c \in F(C)$ such that $F(\delta)(c)=c_{0}$. Thus $\delta:\left(C_{0}, c_{0}\right) \rightarrow(C, c)$ lies in $\mathcal{A}_{\lambda}^{\lambda} / F$, and $\delta\left(f_{1}-f_{2}\right)=\delta \eta \theta=0$, finishing the proof of the first step above.

Finally, an arbitrary $A \in \mathcal{A}_{\kappa}$ is a colimit of objects in $\mathcal{A}_{\kappa}^{\kappa}$, so it is a cokernel of the form $A_{1} \rightarrow A_{0} \rightarrow A \rightarrow 0$ with $A_{1}$ and $A_{0}$ being coproducts of objects in $\mathcal{A}_{\kappa}^{\kappa}$. Using the first step before, we easily get

$$
F(A) \cong \mathcal{A}\left(A, F_{\lambda}\right) \cong \operatorname{colim}_{(X, x) \in \mathcal{A}_{\lambda}^{\lambda} / F} \mathcal{A}(A, X)
$$

canonically.

Remark 1.4. With the notations made in Lemma 1.3 and its proof, the argument used to show the fact that $\mathcal{A}\left(A, F_{\lambda}\right) \cong \operatorname{colim}_{(X, x) \in \mathcal{A}_{\lambda}^{\lambda} / F} \mathcal{A}(A, X)$, for $A=\coprod_{i \in I} U_{i}$, with $U_{i} \in \mathcal{A}_{\kappa}^{\kappa}$, is inspired by [3, Lemma 2.11]. However, we did not only change the settings, 
but we also improved the proof of Franke. A simple translation of his argument in our settings would require the condition card $\mathcal{A}(U, X) \leqslant \lambda$ for all $U \in \mathcal{A}_{\kappa}^{\kappa}$ and all $X \in \mathcal{A}_{\lambda}^{\lambda}$. $A$ priori it is not clear how we may choose such a regular cardinal $\lambda$. Instead, we require $\sum_{U \in \mathcal{C}_{0}} \operatorname{card} \mathcal{A}\left(U, C_{0}\right)<\lambda$, where the left-hand side of this inequality does not depend of $\lambda$.

Recall that we call cofinal a subcategory $\mathcal{S}$ of a category $\mathcal{C}$ that satisfies the following two properties: For every $c \in \mathcal{C}$ there is a map $c \rightarrow s$ in $\mathcal{C}$ for some $s \in \mathcal{S}$, and for any two maps $c \rightarrow s_{1}$ and $c \rightarrow s_{2}$ in $\mathcal{C}$, with $s_{1}, s_{2} \in \mathcal{S}$ there are $s \in \mathcal{S}$ and two maps $s_{1} \rightarrow s$ and $s_{2} \rightarrow s$ in $\mathcal{S}$ such that the composed morphisms $c \rightarrow s_{1} \rightarrow s$ and $c \rightarrow s_{2} \rightarrow s$ are equal. It is well-known that if $\mathcal{S}$ is a cofinal subcategory of $\mathcal{C}$, then colimits over $\mathcal{C}$ and colimits over $\mathcal{S}$ coincide (see [1,0.11]).

Lemma 1.5. Let $\mathcal{A}$ be an abelian category, and let $F: \mathcal{A} \rightarrow \mathcal{A} b$ be a contravariant, exact functor. Let $\mathcal{C} \subseteq \mathcal{A}$ be a subcategory closed under finite coproducts and cokernels. If $\mathcal{S}$ is a subcategory of $\mathcal{C}$ closed under finite coproducts and satisfying the property that every $X \in \mathcal{C}$ admits an embedding $0 \rightarrow X \rightarrow S$ into an object in $\mathcal{S}$, then $\mathcal{S} / F$ is a cofinal subcategory of $\mathcal{C} / F$.

Proof. Let $(X, x) \in \mathcal{C} / F$. Consider an embedding $0 \rightarrow X \stackrel{\alpha}{\rightarrow} S$, with $S \in \mathcal{S}$. Thus $F(S) \stackrel{F(\alpha)}{\longrightarrow} F(X) \rightarrow 0$ is exact, showing that there exists $y \in F(S)$ with $F(\alpha)(y)=x$. Therefore, $\alpha$ is a map in $\mathcal{C} / F$ between $(X, x)$ and $(S, y)$.

Now we claim that if $\alpha: X_{1} \rightarrow X_{2}$ is a map in $\mathcal{C}$, and $x_{2} \in F\left(X_{2}\right)$ is an element with the property $F(\alpha)\left(x_{2}\right)=0$, then there is a morphism $\gamma \in \mathcal{C} / F\left(\left(X_{2}, x_{2}\right),(S, y)\right)$ into an object $(S, y) \in \mathcal{S} / F$ such that $\gamma \alpha=0$. Indeed consider $X$ being defined by exact sequence $X_{1} \stackrel{\alpha}{\rightarrow} X_{2} \stackrel{\beta}{\rightarrow} X \rightarrow 0$. Since the sequence of abelian groups $0 \rightarrow F(X) \stackrel{F(\beta)}{\longrightarrow}$ $F\left(X_{2}\right) \stackrel{F(\alpha)}{\longrightarrow} F\left(X_{1}\right)$ is also exact and $F(\alpha)\left(x_{2}\right)=0$, we obtain an element $x \in F(X)$ such that $F(\beta)(x)=x_{2}$. For obtaining the required $\gamma$, compose $\beta$ with a morphism in $\mathcal{C} / F$ from $(X, x)$ into an object $(S, y)$, which is constructed as in the first part of this proof.

Finally, for two morphisms

$$
\alpha_{1} \in \mathcal{C} / F\left((X, x),\left(S_{1}, y_{1}\right)\right) \text { and } \alpha_{2} \in \mathcal{C} / F\left((X, x),\left(S_{2}, y_{2}\right)\right),
$$

denote by $\rho_{1}$ and $\rho_{2}$ the respective injections of the coproduct $S_{1} \amalg S_{2}$. Then $F\left(\rho_{1} \alpha_{1}-\rho_{2} \alpha_{2}\right)\left(y_{1}, y_{2}\right)=x-x=0$, so our claim for $\alpha=\rho_{1} \alpha_{1}-\rho_{2} \alpha_{2}$ gives a morphism $\left(S_{1} \amalg S_{2},\left(y_{1}, y_{2}\right)\right) \rightarrow(S, y)$ in $\mathcal{C} / F$, with $S \in \mathcal{S}$, such that the composed morphisms $X \rightarrow S_{1} \rightarrow S_{1} \amalg S_{2} \rightarrow S$ and $X \rightarrow S_{2} \rightarrow S_{2} \amalg S_{2} \rightarrow S$ are equal.

Let $\kappa \in \mathfrak{R}$. As usual, a $\kappa$-(co)product means a (co)product of less that $\kappa$ objects. We say that a quasi-locally presentable abelian category $\mathcal{A}$ is weakly $\kappa$-generated if $\mathcal{A}$ coincides with its smallest full subcategory containing $\mathcal{A}_{\kappa}$ and is closed under kernels, cokernels, extensions and $\kappa$-coproducts. We also need the following notation:

$$
\operatorname{Inj}_{\lambda} \mathcal{A}=\left\{S \in \mathcal{A} \mid S \text { is injective and } S \in \mathcal{A}_{\lambda}^{\lambda}\right\} .
$$

Theorem 1.6. Let $\mathcal{A}$ be a quasi-locally presentable, abelian category that is weakly $\kappa$-generated, for some regular cardinal $\kappa$. Suppose also that, for any regular cardinal $\lambda \geqslant \kappa$, every $X \in \mathcal{A}_{\lambda}^{\lambda}$ admits an embedding $0 \rightarrow X \rightarrow S$ into an object $S \in \operatorname{Inj}_{\lambda} \mathcal{A}$. 
Then every exact, contravariant functor $F: \mathcal{A} \rightarrow \mathcal{A} b$ that sends coproducts into products is representable (necessarily by an injective object).

Proof. Fix a contravariant exact functor $F: \mathcal{A} \rightarrow \mathcal{A} b$ that sends coproducts into products. Consider the obvious natural transformation

$$
\phi: \operatorname{colim}_{(X, x) \in \mathcal{A}_{\lambda}^{\lambda} / F} \mathcal{A}(-, X) \rightarrow F .
$$

Since $F$ sends colimits into limits, Lemma 1.3 applies and tells us that there is $\lambda \in \mathfrak{R}$, $\lambda \geqslant \kappa$ such that $\phi$ restricts to an isomorphism

$$
\underset{(X, x) \in \mathcal{A}_{\lambda}^{\lambda} / F}{\operatorname{colim}} \mathcal{A}\left(I_{\kappa}(-), X\right) \cong F I_{\kappa} .
$$

We know that $\mathcal{A}_{\lambda}^{\lambda} / F$ is $\lambda$-filtered (see [5, Korollar 5.4]). Hence colimits of abelian groups indexed over this category are exact and commute with products of less that $\lambda$ objects (see [5, Satz 5.2]). Since every $X \in \mathcal{A}_{\lambda}^{\lambda}$ admits an embedding in an object $S \in \operatorname{Inj}_{\lambda} \mathcal{A}$, we deduce by Lemma 1.5 that $\operatorname{Inj}_{\lambda} \mathcal{A} / F$ is a cofinal subcategory of $\mathcal{A}_{\lambda}^{\lambda} / F$, so

$$
\operatorname{colim}_{(X, x) \in \mathcal{A}_{\lambda}^{\lambda} / F} \mathcal{A}(-, X) \cong \operatorname{colim}_{(S, s) \in \operatorname{Inj}_{\lambda} \mathcal{A} / F} \mathcal{A}(-, S)
$$

is an exact functor. We infer that the full subcategory of $\mathcal{A}$ consisting of all objects $A$ for which $\phi_{A}$ is an isomorphism contains $\mathcal{A}_{\kappa}$ and is closed under kernels, cokernels, extensions and $\kappa$-coproducts (since $\lambda \geqslant \kappa$ ). Therefore, it is equal to $\mathcal{A}$ forced by the hypothesis of weak $\kappa$-generation. This means that $\phi$ is a natural isomorphism, and hence a skeleton of $\mathcal{A}_{\lambda}^{\lambda}$ forms a solution set for $F$. We conclude that $F$ is representable by the general Freyd's adjoint functor theorem.

Example 1.7. The following example shows that the conclusion of Theorem 1.6 requires some kind of weak generation.

Recall that an abelian category is called locally Grothendieck if every set of objects may be included in a subcategory that is Grothendieck (see [13]). Let $K$ be a field. The category $\mathcal{A}=\bigcup_{\lambda \in \mathfrak{R}} \operatorname{Mod}\left(K^{\lambda}\right)$ considered in [13] is locally Grothendieck. By $\operatorname{Mod}\left(K^{\lambda}\right)$ we denote the category of right modules over the ring $K^{\lambda}$. Moreover, the category $\mathcal{A}$ is also quasi-locally presentable. Indeed it is a a big union of a chain of Grothendieck (hence locally presentable) subcategories $\mathcal{A}_{\lambda}=\operatorname{Mod}\left(K^{\lambda}\right)$. For all $\kappa \leqslant \lambda$ in $\mathfrak{R}$ we have $K^{\kappa}=K^{\lambda} e$, where $e=e(\kappa, \lambda) \in K^{\lambda}$ is a central idempotent defined by $e_{\gamma}=1$ for $\gamma \leqslant \kappa$ and 0 otherwise. Thus $K^{\kappa}$ is a direct summand of $K^{\lambda}$, and all $X \in \operatorname{Mod}\left(K^{\lambda}\right)$ decomposes as $X=X e \oplus X(1-e)$. Moreover, for $X, Y \in \operatorname{Mod}\left(K^{\lambda}\right)$, there is no nonzero homomorphisms between $X e$ and $Y(1-e)$. Hence we have

$$
\operatorname{Hom}_{K^{\lambda}}(X, Y)=\operatorname{Hom}_{K^{\kappa}}(X e, Y e) \oplus \operatorname{Hom}_{K^{\lambda}(1-e)}(X(1-e), Y(1-e)) .
$$

Thus we can see $\operatorname{Mod}\left(K^{\kappa}\right)$ as a full split subcategory of $\operatorname{Mod}\left(K^{\lambda}\right)$. We deduce that for every fixed $\kappa \in \mathfrak{R}$ and for every $X \in \mathcal{A}$, there is $\lambda \geqslant \kappa$ such that $X \in \operatorname{Mod}\left(K^{\lambda}\right)$. The assignment $X \mapsto X e$, where $e=e(\kappa, \lambda)$ induces a well defined functor $R_{\kappa}: \mathcal{A} \rightarrow$ $\operatorname{Mod}\left(K^{\kappa}\right)$ that is both the left and the right adjoint of the inclusion functor $I_{\kappa}$, follows by the fact that $\operatorname{Mod}\left(K^{\kappa}\right)$ is a full split subcategory of $\operatorname{Mod}\left(K^{\lambda}\right)$. Thus both the inclusion functor $\operatorname{Mod}\left(K^{\kappa}\right)$ and its right adjoint preserve colimits. 
Using an idea from [10] we may construct a non-representable exact contravariant functor $F: \mathcal{A} \rightarrow \mathcal{A} b$ into products. For every $\lambda \in \mathfrak{R}$, denote by $\lambda^{+}$the successor of $\lambda$ and consider $Q_{\lambda^{+}}$to be an injective cogenerator of $\operatorname{Mod}\left(K^{\lambda^{+}}\right)$. The $K^{\lambda^{+}}$-module $Y_{\lambda}=Q_{\lambda^{+}}(1-e)$, where $e=e\left(\lambda, \lambda^{+}\right)$, is injective and satisfies $\operatorname{Hom}_{K^{\lambda^{+}}}\left(X, Y_{\lambda}\right)=0$ for all $X \in \operatorname{Mod}\left(K^{\lambda}\right)$. The contravariant functor

$$
F: \mathcal{A} \rightarrow \mathcal{A} b, F(X)=\prod_{\lambda \in \mathfrak{R}} \mathcal{A}\left(X, Y_{\lambda}\right)
$$

is well defined. In fact, for $X \in \operatorname{Mod}\left(K^{\kappa}\right)$, we have $\mathcal{A}\left(X, Y_{\lambda}\right)=0$ if $\lambda \geqslant \kappa$, hence $F(X)=\prod_{\lambda<\kappa} \mathcal{A}\left(X, Y_{\lambda}\right)$. Obviously $F$ is exact and sends coproducts into products. But $F$ is not representable, since the strict inclusion of $\operatorname{Mod}\left(K^{\lambda}\right)$ into $\operatorname{Mod}\left(K^{\lambda^{+}}\right)$implies that the cogenerator $Q_{\lambda^{+}}$must contain a nonzero part $Y_{\lambda}$ in $\operatorname{Mod}\left(K^{\lambda^{+}}(1-e)\right)$. The representability of $F$ would mean the existence of the product $Y=\prod_{\lambda \in \mathfrak{R}} Y_{\lambda}$ in $\mathcal{A}$. But this is absurd since $Y$ would have a proper class of endomorphisms, and such objects do not exist in $\mathcal{A}$. Notice that the category $\bigcup_{\lambda \in \mathfrak{R}} \operatorname{Mod}\left(K^{\lambda}\right)$ was used in [13] as an example of a category for which the $\lambda$-pure global dimension is greater than 1 , for all $\lambda \in \mathfrak{R}$. Both this example and our present work have connections with Brown representability. On the other hand, we have

Proposition 1.8. Consider the above locally Grothendieck category

$$
\mathcal{A}=\bigcup_{\lambda \in \Re} \operatorname{Mod}\left(K^{\lambda}\right) .
$$

A contravariant functor $F: \mathcal{A} \rightarrow \mathcal{A} b$ is representable if and only if it sends colimits into limits and there is $\kappa \in \mathfrak{R}$ such that $F \cong F I_{\kappa} R_{\kappa}$.

Proof. If $F \cong \mathcal{A}(-, Y)$ for some $Y \in \mathcal{A}$ then there is $\kappa \in \mathfrak{R}$ such that $Y \in \operatorname{Mod}\left(K^{\kappa}\right)$. Thus for every $X \in \mathcal{A}$, there is $\lambda \geqslant \kappa$ such that $X \in \operatorname{Mod}\left(K^{\lambda}\right)$. Hence

$$
F(X)=\mathcal{A}(X, Y) \cong \mathcal{A}(X e, Y) \cong F I_{\kappa} R_{\kappa}(X) .
$$

Conversely, if $F$ sends colimits into limits then, as in the proof of Lemma 1.3, we obtain $F I_{\kappa} \cong \operatorname{Hom}_{K^{\kappa}}(-, Y)$, for some $Y \in \operatorname{Mod}\left(K^{\kappa}\right)$. Combining this with $F \cong$ $F I_{\kappa} R_{\kappa}$ we deduce:

$$
F \cong \operatorname{Hom}_{K^{\kappa}}\left(R_{\kappa}(-), Y\right) \cong \mathcal{A}\left(-, I_{\kappa}(Y)\right)
$$

therefore $F$ is representable.

Example 1.9. In Theorem 1.6 the exactness of the functor $F: \mathcal{A} \rightarrow \mathcal{A} b$ (which sends coproducts into products) is an essential hypothesis. More precisely, the weaker requirement that $F$ sends colimits into limits is not sufficient to conclude that it is representable. For showing this suppose that the quasi-locally presentable category $\mathcal{A}$ from the Theorem 1.6 is abelian (as in the motivating case of the next section) but is not locally presentable; that is, $\mathcal{A} \neq \mathcal{A}_{\lambda}$ for every $\lambda \in \mathfrak{R}$. The fact that $\mathcal{A}$ is weakly generated, which is used in combination with the exactness of $F$, does not play any role in this example. The exactness of $R_{\lambda}$ implies that $\mathcal{A}_{\lambda}$ is equivalent to quotient category of $\mathcal{A}$ modulo the Serre subcategory $\operatorname{Ker} R_{\lambda}=\left\{X \in \mathcal{A} \mid R_{\lambda}(X)=0\right\}$. But $R_{\lambda}$ is not an equivalence, forcing $\operatorname{Ker} R_{\lambda} \neq 0$. Consider $0 \neq X_{\lambda} \in \mathcal{A}$ such that 
$R_{\lambda}\left(X_{\lambda}\right)=0$, for every $\lambda \in \mathfrak{R}$. Strictly speaking we need here a version of axiom of choice that works for proper classes. As in Example 1.7, we infer that the functor

$$
F=\prod_{\lambda \in \Re} \mathcal{A}\left(-, X_{\lambda}\right)
$$

is well defined since for every $X \in \mathcal{A}$ we have $X \in \mathcal{A}_{\kappa}$ for some $\kappa \in \mathfrak{R}$, so $\mathcal{A}\left(X, X_{\lambda}\right)$ $=0$ for all $\lambda \geqslant \kappa$. It is easy to see that this functor does the job we claim.

\section{The abelianization of a well generated triangulated cate- gory}

The main purpose of this section is to show that the abelianization of a triangulated category that is well generated in the sense of Neeman is quasi-locally presentable and satisfies the hypothesis of Theorem 1.6. Consequently we obtain a new proof of Brown representability theorem for such triangulated categories.

Consider a preadditive category $\mathcal{T}$. By a $\mathcal{T}$-module we understand a functor $X: \mathcal{T}^{\mathrm{op}} \rightarrow \mathcal{A} b$. Such a functor is called finitely presentable if there is an exact sequence of functors

$$
\mathcal{T}(-, y) \rightarrow \mathcal{T}(-, x) \rightarrow X \rightarrow 0
$$

for some $x, y \in \mathcal{T}$. Using Yoneda lemma, we know that the class of all natural transformations between two $\mathcal{T}$-modules $X$ and $Y$ denoted $\operatorname{Hom}_{\mathcal{T}}(X, Y)$ is actually a set, provided that $X$ is finitely presentable. We consider the category $\bmod (\mathcal{T})$ of all finitely presentable $\mathcal{T}$-modules, having $\operatorname{Hom}_{\mathcal{T}}(X, Y)$ as morphisms spaces, for all $X, Y \in \bmod (\mathcal{T})$. The Yoneda functor

$$
H=H_{\mathcal{T}}: \mathcal{T} \rightarrow \bmod (\mathcal{T}) \text { given by } H_{\mathcal{T}}(x)=\mathcal{T}(-, x)
$$

is an embedding of $\mathcal{T}$ into $\bmod (\mathcal{T})$, according to Yoneda lemma. If, in addition, $\mathcal{T}$ has coproducts then $\bmod (\mathcal{T})$ is cocomplete and the Yoneda embedding preserves coproducts. It is also well-known (and easy to prove) that, if $F: \mathcal{T} \rightarrow \mathcal{A}$ is a functor into an additive category with cokernels, then there is a unique, up to a natural isomorphism, right exact functor $F^{*}: \bmod (\mathcal{T}) \rightarrow \mathcal{A}$, such that $F=F^{*} H_{\mathcal{T}}$ (see $[\mathbf{8}$, Lemma A.1]). Moreover, $F$ preserves coproducts if and only if $F^{*}$ preserves colimits.

In this section the category $\mathcal{T}$ will be triangulated with splitting idempotents. For the definition and basic properties of triangulated categories the standard reference is [11]. Note that $\mathcal{T}$ has splitting idempotents, provided that $\mathcal{T}$ has countable coproducts, according to [11, Proposition 1.6.8]. Recall that $\mathcal{T}$ is supposed to be additive. A functor $\mathcal{T} \rightarrow \mathcal{A}$ into an abelian category $\mathcal{A}$ is called homological if it sends triangles into exact sequences. A contravariant functor $\mathcal{T} \rightarrow \mathcal{A}$ that is homological when regarded as a functor $\mathcal{T}^{\mathrm{op}} \rightarrow \mathcal{A}$ is called cohomological (see [11, Definition 1.1.7 and Remark 1.1.9]). An example of a homological functor is the Yoneda embedding $H_{\mathcal{T}}: \mathcal{T} \rightarrow \bmod (\mathcal{T})$. We know that $\bmod (\mathcal{T})$ is an abelian category, and for every functor $F: \mathcal{T} \rightarrow \mathcal{A}$ into an abelian category, the unique right exact functor $F^{*}: \bmod (\mathcal{T}) \rightarrow \mathcal{A}$ extending $F$ is exact if and only if $F$ is homological, by [6, Lemma 2.1]. This is the reason for which $\bmod (\mathcal{T})$ is called the abelianization of the triangulated category $\mathcal{T}$ and is sometimes denoted by $\mathrm{A}(\mathcal{T})$. By [11, Corollary 5.1.23], $\mathrm{A}(\mathcal{T})$ 
is a Frobenius abelian category with enough injectives and enough projectives that are, up to isomorphism, exact objects of the form $\mathcal{T}(-, x)$ for some $x \in \mathcal{T}$.

A first link between representability of functors defined on $\mathcal{T}$, respectively on $\mathrm{A}(\mathcal{T})$ is given by:

Lemma 2.1. If $\mathcal{T}$ is a triangulated category with splitting idempotents, then a cohomological functor $F: \mathcal{T} \rightarrow \mathcal{A} b$ is representable if and only if its extension $F^{*}: \mathrm{A}(\mathcal{T}) \rightarrow$ $\mathcal{A} b$ is representable.

Proof. The cohomological functor $F: \mathcal{T} \rightarrow \mathcal{A} b$ can be interpreted as a homological functor $\mathcal{T} \rightarrow \mathcal{A} b^{\mathrm{op}}$ that has a unique extension to $\mathrm{A}(\mathcal{T})$. Therefore, $F$ extends uniquely to a contravariant, exact functor $F^{*}: \mathrm{A}(\mathcal{T}) \rightarrow \mathcal{A} b$, defined as $F^{*} \cong \operatorname{Hom}_{\mathcal{T}}(-, F)$. We recall that Hom denotes the set of all natural transformations, and it coincides with the morphisms spaces in $\mathrm{A}(\mathcal{T})$ only if $F \in \mathrm{A}(\mathcal{T})$.

If $F$ is representable, then $F \in \mathrm{A}(\mathcal{T})$, and $F^{*}$ is represented by $F$. Conversely if $F^{*}$ is representable by an object in $\mathrm{A}(\mathcal{T})$, then this object must be isomorphic to $F$; therefore $F \in \mathrm{A}(\mathcal{T})$. Because $F^{*}$ is exact, $F$ must be injective, hence representable.

We say that $\mathcal{T}$ satisfies the Brown representability theorem if every cohomological functor $F: \mathcal{T} \rightarrow \mathcal{A} b$ that sends coproducts into products is representable. Then we record:

Corollary 2.2. Let $\mathcal{T}$ be a triangulated category with coproducts. The following are equivalent:

(i) $\mathcal{T}$ satisfies Brown representability theorem.

(ii) Every exact contravariant functor $F: \mathrm{A}(\mathcal{T}) \rightarrow \mathcal{A} b$ that sends coproducts into products is representable.

(iii) Every exact covariant functor $F: \mathrm{A}(\mathcal{T}) \rightarrow \mathcal{A}$ that preserves colimits, having values into an abelian cocomplete category with enough injectives, has a right adjoint.

Proof. The equivalence (i) $\Leftrightarrow$ (ii) follows by Lemma 2.1, whereas the implication (iii) $\Rightarrow$ (ii) is obvious, by replacing contravariant functors $\mathrm{A}(\mathcal{T}) \rightarrow \mathcal{A} b$ with covariant functors $\mathrm{A}(\mathcal{T}) \rightarrow \mathcal{A} b^{\mathrm{op}}$. Finally, (i) $\Rightarrow$ (iii) follows by [2, Theorem 1.1].

Let $\mathcal{T}$ is a triangulated category with coproducts. We need the following definitions: For a regular cardinal $\lambda$, a $\lambda$-localizing subcategory of $\mathcal{T}$ is a triangulated subcategory closed under $\lambda$-coproducts. A localizing subcategory is a subcategory that is $\lambda$-localizing for all $\lambda$. Consider a set of objects $\mathcal{S} \subseteq \mathcal{T}$ that is closed under suspensions and desuspensions. We say that $\mathcal{T}$ is generated (in the triangulated sense) by $\mathcal{S}$, provided that an object $t \in \mathcal{T}$ vanishes, whenever $\mathcal{T}(s, t)=0$ for all $s \in \mathcal{S}$. Further, we say that $\mathcal{T}$ is perfectly generated by the set of objects $\mathcal{S}$ if $\mathcal{S}$ generates $\mathcal{T}$, and, for any $s \in \mathcal{S}$, the map $\mathcal{T}\left(s, \coprod_{i \in I} x_{i}\right) \rightarrow \mathcal{T}\left(s, \coprod_{i \in I} y_{i}\right)$ is surjective, for every set of maps $\left\{x_{i} \rightarrow y_{i} \mid i \in I\right\}$ such that $\mathcal{T}\left(s, x_{i}\right) \rightarrow \mathcal{T}\left(s, y_{i}\right)$ is surjective, for all $i \in I$. Finally, $\mathcal{T}$ is called well $\lambda$-generated, where $\lambda \in \mathfrak{R}$, provided that $\mathcal{T}$ is perfectly generated by a set of objects, which are also $\lambda$-small, that is, every map $s \rightarrow \coprod_{i \in I} x_{i}$, with $s \in \mathcal{S}$, factors trough a coproduct $\coprod_{i \in I^{\prime}} x_{i}$ with card $I^{\prime}<\lambda$; the category $\mathcal{T}$ is well generated if it is 
well $\lambda$-generated, for some $\lambda$. Following $[\mathbf{7}$, Theorem A], this definition is equivalent to the original one given by Neeman. Note that, by [9, Corollary 2.6], if $\mathcal{T}$ is perfectly generated by $\mathcal{S}$, then $\mathcal{T}$ coincides with its smallest $\aleph_{1}$-localizing subcategory that contains arbitrary coproducts of objects in $\mathcal{S}$.

A category $\mathcal{C}$ is called $\lambda$-cocomplete if $\mathcal{C}$ has $\lambda$-coproducts and cokernels. It is easy to see that $\mathcal{C}$ is $\lambda$-cocomplete if and only if it contains all colimits of diagrams with less that $\lambda$ morphisms. A $\mathcal{C}$-module over a $\lambda$-cocomplete category is called $\lambda$-left exact if it is left exact and sends $\lambda$-coproducts into products. Provided that the category $\mathcal{C}$ is essentially small, the class $\operatorname{Hom}_{\mathcal{C}}(X, Y)$ is actually a set for all $\mathcal{C}$-modules $X, Y$. Thus we are allowed to consider the category $\operatorname{Mod}(\mathcal{C})$ of all $\mathcal{C}$-modules. If $\mathcal{C}$ is also $\lambda$-cocomplete, then denote by $\operatorname{Lex}_{\lambda}\left(\mathcal{C}^{\mathrm{op}}, \mathcal{A} b\right)$ the full subcategory of $\operatorname{Mod}(\mathcal{C})$ consisting of $\lambda$-left exact modules. We know that $\operatorname{Lex}_{\lambda}\left(\mathcal{C}^{\mathrm{op}}, \mathcal{A} b\right)$ is a locally $\lambda$-presentable category, and the embedding $\mathcal{C} \rightarrow \operatorname{Lex}_{\lambda}\left(\mathcal{C}^{\text {op }}, \mathcal{A} b\right)$ given by $X \mapsto \mathcal{C}(-, X)$ identifies $\mathcal{C}$, up to isomorphism, with the subcategory of $\lambda$-presentable objects in $\operatorname{Lex}_{\lambda}\left(\mathcal{C}^{\mathrm{op}}, \mathcal{A} b\right)$ (see [5, Korollar 7.9]).

As before, let $\lambda$ denote a regular cardinal. If $\mathcal{S}$ is a preadditive, essentially small category with $\lambda$-coproducts, then denote by $\operatorname{Prod}_{\lambda}\left(\mathcal{S}^{\mathrm{op}}, \mathcal{A} b\right)$ the full subcategory of $\operatorname{Mod}(\mathcal{S})$, consisting of those modules that preserve $\lambda$-products. Clearly a finitely presentable $\mathcal{S}$-module, that is, an element in $\bmod (\mathcal{S})$, preserves arbitrary products, hence it belongs to $\operatorname{Prod}_{\lambda}\left(\mathcal{S}^{\mathrm{op}}, \mathcal{A} b\right)$.

Lemma 2.3. For a regular cardinal $\lambda$, consider an additive, essentially small category $\mathcal{S}$ having $\lambda$-coproducts. Then $\operatorname{Prod}_{\lambda}\left(\mathcal{S}^{\mathrm{op}}, \mathcal{A} b\right)$ is a locally $\lambda$-presentable category, and the embedding $\bmod (\mathcal{S}) \stackrel{\subseteq}{\rightarrow} \operatorname{Prod}_{\lambda}\left(\mathcal{S}^{\text {op }}, \mathcal{A} b\right)$ identifies $\bmod (\mathcal{S})$ with the full subcategory of $\operatorname{Prod}_{\lambda}\left(\mathcal{S}^{\mathrm{op}}, \mathcal{A} b\right)$ consisting of all $\lambda$-presentable objects.

Proof. The category $\bmod (\mathcal{S})$ has obviously $\lambda$-coproducts and cokernels, so it is $\lambda$-cocomplete. According to [8, Lemma B.1], there is an equivalence of categories

$$
\operatorname{Lex}_{\lambda}\left(\bmod (\mathcal{S})^{\mathrm{op}}, \mathcal{A} b\right) \rightarrow \operatorname{Prod}_{\lambda}\left(\mathcal{S}^{\mathrm{op}}, \mathcal{A} b\right), X \mapsto X H_{\mathcal{S}}
$$

where $H_{\mathcal{S}}: \mathcal{S} \rightarrow \bmod (\mathcal{S})$ denotes the Yoneda functor. Thus $\operatorname{Prod}_{\lambda}\left(\mathcal{S}^{\mathrm{op}}, \mathcal{A} b\right)$ is locally $\lambda$-presentable. Further, the identification of $\lambda$-presentable objects in $\operatorname{Prod}_{\lambda}\left(\mathcal{S}^{\mathrm{op}}, \mathcal{A} b\right)$ follows by discussion above concerning $\lambda$-presentable objects in $\operatorname{Lex}_{\lambda}\left(\mathcal{C}^{\mathrm{op}}, \mathcal{A} b\right)$.

Suppose now that $\mathcal{T}$ is well $\kappa$-generated triangulated category, having a perfectly generating set $\mathcal{S}$ consisting of $\kappa$-small objects. For any $\lambda \geqslant \kappa$, we consider the smallest $\lambda$ localizing subcategory of $\mathcal{T}$ that contains $\mathcal{S}$ and denote it by $\mathcal{T}^{\lambda}$. The objects in $\mathcal{T}^{\lambda}$ are called $\lambda$-compact. By [7, Lemma 5] the category of $\lambda$-compact objects in $\mathcal{T}$ is independent of $\mathcal{S}$. Clearly it is essentially small and a skeleton of $\mathcal{T}^{\lambda}$ generates $\mathcal{T}$. Moreover $\mathcal{T}^{\lambda}$ has $\lambda$-coproducts. Denote $\mathrm{A}_{\lambda}(\mathcal{T})=\operatorname{Prod}_{\lambda}\left(\left(\mathcal{T}^{\lambda}\right)^{\mathrm{op}}, \mathcal{A} b\right)$, for $\lambda \geqslant \kappa$ and $\mathrm{A}_{\lambda}(\mathcal{T})=0$ otherwise. We know by [11, Proposition A.1.8] that $\mathrm{A}_{\lambda}(\mathcal{T})$ is locally $\lambda$-presentable, and by $\left[\mathbf{1 1}\right.$, Proposition 6.5.3] that the restriction functor $R_{\lambda}: \mathrm{A}(\mathcal{T}) \rightarrow$ $\mathrm{A}_{\lambda}(\mathcal{T})$ has a fully faithful left adjoint $I_{\lambda}: \mathrm{A}_{\lambda}(\mathcal{T}) \rightarrow \mathrm{A}(\mathcal{T})$, therefore we may identify $\mathrm{A}_{\lambda}(\mathcal{T})$ to a coreflective subcategory of $\mathrm{A}(\mathcal{T})$.

Proposition 2.4. Fix a regular cardinal $\kappa>\aleph_{0}$. If $\mathcal{T}$ is a well $\kappa$-generated triangulated category, then $\mathrm{A}(\mathcal{T})$ is a quasi-locally presentable abelian category that is weakly $\kappa$-generated. 
Proof. Denote by $\mathcal{A}$ the smallest subcategory of $\mathrm{A}(\mathcal{T})$ that is closed under kernels, cokernels, extensions, countable coproducts and contains $\mathrm{A}_{\kappa}(\mathcal{T})$. Let us show that $\mathrm{A}(\mathcal{T})=\mathcal{A}$. Observe first that if $T \rightarrow U \rightarrow X \rightarrow Y \rightarrow Z$ is an exact sequence with $T, U, Y, Z \in \mathcal{A}$ then we can construct the commutative diagram with exact rows and column

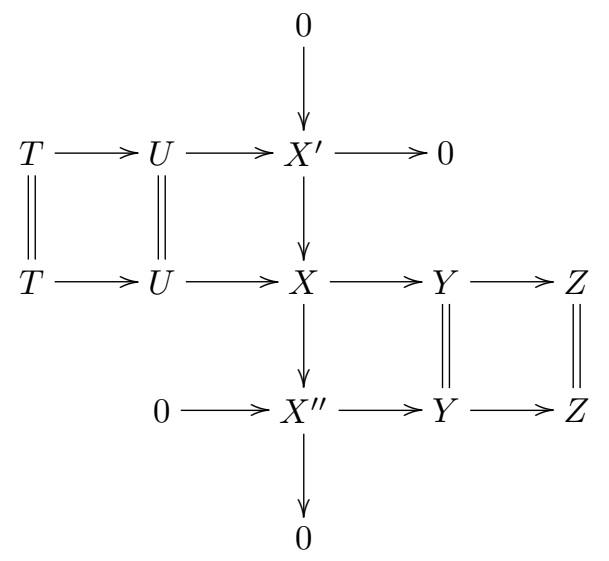

showing that $X \in \mathcal{A}$. Therefore, if $x \rightarrow y \rightarrow z \rightsquigarrow$ is a triangle in $\mathcal{T}$ with $H(x), H(z) \in$ $\mathcal{A}$ then $H(y) \in \mathcal{A}$. It is shown in [9, Theorem 2.5] that every object $x \in \mathcal{T}$ is isomorphic to a homotopy colimit of a tower $x^{0} \rightarrow x^{1} \rightarrow \cdots$ such that $x^{0}=0$ and for every $n \in \mathbb{N}$ we have a triangle $p_{n} \rightarrow x^{n} \rightarrow x^{n+1} \rightsquigarrow$ with $p_{n}$ being a coproduct of objects in $\mathcal{T}^{\kappa}$. Inductively, $H\left(x^{n}\right) \in \mathcal{A}$, for all $n \in \mathbb{N}$. Hence $H\left(\coprod_{n \in \mathbb{N}} x^{n}\right) \cong \coprod_{n \in \mathbb{N}} H\left(x^{n}\right) \in \mathcal{A}$, and finally, $H(x) \in \mathcal{A}$. Now, for every $X \in \mathrm{A}(\mathcal{T})$ there is an exact sequence $H(y) \rightarrow$ $H(x) \rightarrow X \rightarrow 0$, with $x, y \in \mathcal{T}$, thus $X \in \mathcal{A}$.

Note that we have already shown that $\mathcal{T}$ coincides with its smallest $\aleph_{1}$-localizing subcategory that contains a skeleton of $\mathcal{T}^{\kappa}$. Therefore, the proof of [11, Proposition 8.4.2] (more precisely $[\mathbf{1 1}, 8.4 .2 .3]$ ) works for our case. Hence $\mathcal{T}=\bigcup_{\lambda \geqslant \kappa} \mathcal{T}^{\lambda}$, and further $\mathrm{A}(\mathcal{T})=\bigcup_{\lambda \in \mathfrak{R}} \mathrm{A}_{\lambda}(\mathcal{T})$. In addition an immediate consequence of Lemma $[\mathbf{1 1}$, 6.5.1] is that the right adjoint of the inclusion functor $\mathrm{A}_{\lambda}(\mathcal{T}) \rightarrow \mathrm{A}(\mathcal{T})$ preserves colimits, and all conditions from the definition of a weakly $\kappa$-generated quasi-locally presentable category are fulfilled.

Theorem 2.5. If $\mathcal{T}$ is a well generated triangulated category, then every functor $F: \mathrm{A}(\mathcal{T}) \rightarrow \mathcal{A} b$ that is contravariant, exact and sends coproducts into products is representable.

Proof. Without losing the generality we may assume that $\mathcal{T}$ is well $\kappa$-generated, for some $\kappa \geqslant \aleph_{1}$ (if not, we replace $\kappa$ by $\aleph_{1}$ ). By Proposition 2.4, $\mathrm{A}(\mathcal{T})$ is a weakly $\kappa$ generated quasi-locally presentable category. In order to apply Theorem 1.6, we have only to show that every $\lambda$-presentable object $X$ of $\mathrm{A}_{\lambda}(\mathcal{T})$ admits an embedding into an object in $S \in \mathrm{A}_{\lambda}(\mathcal{T})$ that is $\lambda$-presentable in $\mathrm{A}_{\lambda}(\mathcal{T})$ and injective in $\mathrm{A}(\mathcal{T})$. But this follows immediately from Lemma 2.3, since, according to [11, Corollary 5.1.23], every $X \in \bmod \left(\mathcal{T}^{\lambda}\right)$ admits an embedding into an object of the form $H(x)$ with $x \in \mathcal{T}^{\lambda}$.

Note that the category $\mathrm{A}(\mathcal{T})$ is usually "huge", in the sense that it is not well (co)powered, as we learned on [11, Appendix C]. Thus Proposition 2.4 and 
Theorem 2.5 provide an example of such a huge category, which is quasi-locally presentable and for which representability Theorem 1.6 applies.

Combining Theorem 2.5 with Corollary 2.2 we obtain a new proof for:

Corollary 2.6. Every well generated triangulated categories satisfies Brown representability theorem.

Example 2.7. Recall from [12] the definition: A triangulated category with coproducts is called locally well generated, provided that every localizing subcategory that is generated (in the triangulated sense) by a set of objects is well generated. The typical example of a locally well generated triangulated category, which is not well generated, is the homotopy category $\mathbf{K}(\operatorname{Mod} R)$ where $R$ is a ring that is not pure-semisimple (see [12, Theorem 3.5]). Objects in this category are complexes of $R$-modules, and maps are classes of homotopy equivalent maps of complexes.

Let consider $R=\mathbb{Z}$, so $\mathcal{T}=\mathbf{K}(\mathcal{A} b)$ is locally well generated, but not well generated. Then we want to construct a non-representable exact contravariant functor $F: \mathrm{A}(\mathbf{K}(\mathcal{A} b)) \rightarrow \mathcal{A} b$ that sends coproducts into products. For this purpose, observe that there are objects $Y_{\lambda} \in \mathbf{K}(\mathcal{A} b)$ with $\lambda \in \mathfrak{R}$ such that the functor:

$$
F=\prod_{\lambda \in \mathfrak{R}} \mathcal{T}\left(-, Y_{\lambda}\right): \mathbf{K}(\mathcal{A} b) \rightarrow \mathcal{A} b
$$

is cohomological, sends coproducts into products but is not representable, as it may be seen in [10, Example 11]. Note that the argument showing that this functor is well defined is similar to the one used in Examples 1.7 and 1.9. By Lemma 2.1 the functor

$$
F^{*}: \mathrm{A}(\mathbf{K}(\mathcal{A} b)) \rightarrow \mathcal{A} b, F^{*}(X)=\operatorname{Hom}_{\mathbf{K}(\mathcal{A} b)}\left(X, \prod_{\lambda \in \mathfrak{R}} \mathcal{T}\left(-, Y_{\lambda}\right)\right)
$$

is contravariant, exact, and sends coproducts into products, but it is not representable.

\section{References}

[1] J. Adámek and J. Rosický, Locally presentable and accessible categories, London Math. Soc. Lecture Notes Ser. 187, Cambridge University Press, Cambridge, 1994.

[2] S. Breaz and G.C. Modoi, A reformulation of Brown representability theorem, Mathematica (Cluj) 51 (2009), no. 2, 129-133.

[3] J. Franke, On the Brown representability theorem for triangulated categories, Topology 40 (2001), no. 4, 667-680.

[4] P. Freyd, Abelian categories. An introduction to the theory of functors, Harper \& Row, New York, 1964.

[5] P. Gabriel and F. Ulmer, Lokal präsentierbare Kategorien, Springer Lecture Notes in Math. 221, Springer-Verlag, New York, 1971.

[6] H. Krause, Smashing subcategories and the telescope conjecture - an algebraic approach, Invent. Math. 139 (2000), no. 1, 99-133.

[7] H. Krause, On Neeman's well generated triangulated categories, Documenta Math. 6 (2001), 121-126. 
[8] H. Krause, Localization theory for triangulated categories, in Triangulated categories, London Math. Soc. Lecture Note Ser. 375 (2010), 161-235, Cambridge, Univ. Press, Cambridge.

[9] G.C. Modoi, On perfectly generating projective classes in triangulated categories, Comm. Algeba, 38 (2010), no. 3, 995-1011.

[10] G.C. Modoi and J. Šťvíček, Brown representability often fails for homotopy categories of complexes, J. K-Theory 9 (2012), 151-160.

[11] A. Neeman, Triangulated categories, Annals of Mathematics Studies 148, Princeton University Press, Princeton, NJ, 2001.

[12] J. Šťvíček, Locally well generated homotopy categories of complexes, Documenta Math. 15 (2010), 507-525.

[13] J. Trlifaj, Brown representability test problems for locally Grothendieck categories, Appl. Cat. Struct. 20 (2012), no. 1, 97-102.

George Ciprian Modoi cmodoi@math.ubbcluj.ro

Faculty of Mathematics and Computer Science, Babeş-Bolyai University, RO-400084 Cluj-Napoca, Romania 